\title{
Analysis of the miRNA and mRNA involved in osteogenesis of adipose-derived mesenchymal stem cells
}

\author{
BO JIA $^{1}$, ZHAOQIANG ZHANG $^{1}$, XIAOLING QIU ${ }^{1}$, HONGXING CHU $^{1}$, \\ XIANG SUN ${ }^{1}$, XIANGHUAI ZHENG ${ }^{1}$, JIANJIANG ZHAO ${ }^{1}$ and ${\text { QIN } \mathrm{LI}^{2}}^{2}$ \\ ${ }^{1}$ Laboratory for Oral Diseases, Department of Oral and Maxillofacial Surgery, Stomatological Hospital, Southern Medical \\ University, Guangzhou, Guangdong 510280; ${ }^{2}$ Guangzhou School of Clinical Medicine, Southern Medical University, \\ Guangzhou General Hospital of Guangzhou Military Region, Guangzhou, Guangdong 510010, P.R. China
}

Received November 17, 2017; Accepted March 29, 2018

DOI: $10.3892 /$ etm.2018.6303

\begin{abstract}
Mesenchymal stem cells (MSCs) are bone marrow stromal cells capable of differentiating into different tissue types. Osteoblastic differentiation is a complex process that is critical for bone formation. An increasing number of studies have suggested that microRNAs (miRNAs) may serve important roles in various biological processes, including osteogenesis of MSCs. However, less is known about the participation of particular miRNAs in the osteogenic differentiation of adipose-derived stem cells (ADSCs). In order to identify functional miRNAs and the key genes involved in the osteogenesis of MSCs, the present study reconstructed a global network using data from the National Center for Biotechnology Information Gene Expression Omnibus. Meanwhile, gene ontology and pathway analysis were performed using the Cytoscape plug-in BinGO and the Database for Annotation, Visualization, and Integration Discovery, respectively. An miRNA-mRNA network composed of 72 mRNA and nine miRNA nodes advised by bioinformatics analysis was constructed. These mRNAs and miRNAs were predicted to be involved in the regulation of osteogenic differentiation of ADSCs according to the gene microarray. In the present study, six miRNAs (miR-143-3p, miR-135a-5p, miR-31-5p, miR-22-3p, miR-193b-3p and let-7i-5p) were observed to be highly associated with the
\end{abstract}

Correspondence to: Professor Jianjiang Zhao, Laboratory for Oral Diseases, Department of Oral and Maxillofacial Surgery, Stomatological Hospital, Southern Medical University, 366 South Jiang Nan Road, Guangzhou, Guangdong 510280, P.R. China E-mail: jianjiangzhao123@sina.com

Professor Qin Li, Guangzhou School of Clinical Medicine, Southern Medical University, Guangzhou General Hospital of Guangzhou Military Region, 111 Liu Hua Road, Yue Xiu, Guangzhou, Guangdong 510010, P.R. China

E-mail: liqin19880@163.com

Key words: osteogenic differentiation, adipose-derived stem cells, microarray, dihydropyrimidinase like 3 osteogenesis of ADSCs, and dihydropyrimidinase like 3 was identified as a novel regulator in this process. These results provide support for further investigations into the management of bone regeneration-associated diseases.

\section{Introduction}

Bone is an organ that has the potential for persistent regeneration into adult life and is the only tissue that undergoes constant remodeling throughout life (1). Efficacious bone regeneration could influence the management of bone and musculoskeletal-associated disorders $(2,3)$. While most fractures recover spontaneously, problems in this regenerative process may result in a variety of diseases.

Mesenchymal stem cells (MSCs) are non-hematopoietic stem cells, which have the ability to differentiate into tissues of mesenchymal, as well as non-mesenchymal origin. For example, MSCs are able to differentiate into osteoblastic, chondrogenic and adipogenic lineages (4-6). In the last few years, MSCs have attracted increasing attention for their potential use in identifying the differentiation signaling pathways, facilitating tissue engineering and in their roles as gene vectors and immunomodulators in autoimmune diseases (7-9). In addition to bone marrow, MSCs have been successfully isolated from other tissues, such as adipose-derived mesenchymal stem cells (ADSCs) $(10,11)$. The osteogenesis of ADSCs is a complex process, which is controlled by a series of endogenous and environmental factors and signaling pathways $(12,13)$. However, its effect and the underlying mechanisms involved in the process of bone regeneration remains unclear.

Although thousands of microRNAs (miRNAs) and mRNAs have been identified and deposited in several public databases, such as GENCODE (https://www.gencodegenes. org/), NONCODE (http://www.noncode.org/) and LNCipedia (https://ncipedia.org/), the functional characterization of these is still in its initial stages. So far, only a number of miRNAs and mRNAs have been functionally well explored. It is hypothesized that functionally associated miRNAs and mRNAs may often be involved in several physiological processes; however, their involvement in the osteogenesis of ADSCs has not yet been completely investigated. 
Since miRNAs regulate gene expression via post-transcriptional inhibition or degradation of mRNAs, the potential interaction between differentially expressed miRNAs and mRNAs in the ADSC-derived osteoblasts was analyzed. In the current study, a global network using data from the National Center for Biotechnology Information Gene Expression Omnibus (NCBI GEO, https://www.ncbi.nlm.nih. gov/geo/) identified numerous miRNAs and potential mRNA targets, which were predicted to participate in the osteogenic differentiation of ADSCs. Furthermore, dihydropyrimidinase like 3 (DPYSL3), a novel key regulator of osteogenic differentiation, was identified, which may present a potential therapeutic target in the management of bone regeneration-associated diseases.

\section{Materials and methods}

Raw data. GEO is a public functional genomics data repository supporting minimum information about a microarray experiment (MIAME)-compliant data submissions. Tools are designed to query and download gene expression profiles for further research. In the present study, human miRNA expression data based on the Agilent-031181 Unrestricted_ Human_miRNA_V16.0_Microarray was downloaded from NCBI GEO (GSE72429) and alterations in the miRNA expression profile during osteogenic differentiation were analyzed in human ADSCs using a microarray-based approach. In addition, human mRNA data based on the [HuGene-1_1-st] Affymetrix Human Gene 1.1 ST Array was downloaded from NCBI GEO (GSE37329) with the purpose of screening for the genes involved in the osteogenic differentiation of ADSCs.

Screening of differentially expressed miRNAs (DEMis) and mRNAs (DEMs). Two sets of microarrays were used to identify DEMis and DEMs following the osteogenesis of ADSCs. The Benjamini and Hochberg (using the R package 'limma') (14) method was used to adjust the P-value for the correction of false positive outputs, while $\log \mathrm{FC}$ was used to represent the fold change of down- or upregulated genes in ADSCs prior to and following the induction of osteogenic differentiation. The DEMis and DEMs were selected when $\mathrm{P}<0.05$ and $\mid \log \mathrm{FCl}>1.5$.

Prediction of target mRNAs of DEMis. The target genes of the DEMis from GSE72429 were predicted using TargetScan (http://www.targetscan.org), an online database for predicting miRNA targets (15). The predicted target genes were aligned with the DEMs to obtain an intersection for further analysis.

Functional and pathway enrichment analysis. To assess functional enrichment, gene ontology (GO) Biological Processes term and Kyoto Encyclopedia of Genes and Genomes (KEGG, http://www.genome.jp/kegg/) pathway analyses of mRNAs in the miRNA-mRNA network were performed using the Database for Annotation, Visualization, and Integration Discovery (DAVID, https://david.ncifcrf.gov/). GO and KEGG pathway enrichment analysis for DEMis was performed using the Functional Enrichment analysis tool (FunRich, http://www. funrich.org/), a stand-alone software tool used primarily for functional enrichment and interaction network analysis of genes and proteins. These analyses provide a comprehensive set of functional annotation tools for investigators to understand the biological meaning behind large lists of genes (16).

Protein-protein interaction (PPI) network and module selection. The STRING database (http://string-db.org/) was used for the analysis of PPIs, including direct and indirect associations (17), and Cytoscape (http://www.cytoscape.org/) was used for the visual exploration of biomolecule interaction networks (18) The DEMs were mapped in STRING to evaluate the PPI information and visualized using Cytoscape.

Cell culture, induction of differentiation and transfection. Human ADSCs were purchased from ScienCell Research Laboratories, Inc. (San Diego, CA, USA) and routinely maintained in Dulbecco's modified Eagle's medium with high glucose (Thermo Fisher Scientific, Inc., Waltham, MA, USA) supplemented with $10 \%$ fetal bovine serum (GE Healthcare Life Sciences, Logan, UT, USA) at $37^{\circ} \mathrm{C}$ in a humidified atmosphere of $5 \% \mathrm{CO}_{2}$. The culture medium was refreshed every other day. For differentiation, the cells were cultured in osteogenic medium consisting of standard growth medium supplemented with $10 \mathrm{mM}$ dexamethasone, $0.2 \mathrm{mM}$ L-ascorbic acid, $10 \mathrm{mM} \beta$-glycerophosphate and $10 \mathrm{mM}$ 1,25-dihydroxyvitamin D3 (Sigma-Aldrich; Merck KGaA, Darmstadt, Germany). Cells cultured in a normal medium were used as a control. Cells were then seeded in 6 -well plates at a density of $2 \times 10^{5}$ cells $/ \mathrm{ml}$ per well and transfection was subsequently performed using Lipofectamine 3000 (Thermo Fisher Scientific, Inc.) according to the manufacturer's instructions. After $48 \mathrm{~h}$ of transfection, the cells were collected and used for further experimentation. The short hair RNA plasmids (pGPU6/sh-GFP, pGPU6/sh-DPYSL3-1 and pGPU6/sh-DPYSL3-2) and overexpression lentivruses (LV/GFP and LV/DPYSL) were chemically synthesized by Shanghai GenechemCo., Ltd., (Shanghai, China).

Reverse transcription-quantitative polymerase chain reaction $(R T-q P C R)$. Tripure isolation reagent (Invitrogen; Thermo Fisher Scientific, Inc.) was used for the extraction of total RNA from cell-monolayers, according to the manufacturer's instructions. The first cDNA strands were synthesized using oligodT primers and Revoscript ${ }^{\mathrm{TM}}$ Reverse Transcription PreMix (Intron Biotechnology, Inc., Seongnam, Korea). Analysis of the expressed mRNA was performed using Brilliant II SYBR ${ }^{\circledR}$ Green QPCR Master Mix (Agilent Technologies, Inc., Santa Clara, CA, USA) and a Light Cycler Nano Machine (Roche Applied Science, Rotkreuz, Switzerland). A 35 cycle-thermal program was conducted, consisting of denaturation at $95^{\circ} \mathrm{C}$ for $15 \mathrm{sec}$, annealing at $90^{\circ} \mathrm{C}$ for $15 \mathrm{sec}$ and extension at $72^{\circ} \mathrm{C}$ for $15 \mathrm{sec}$. The primer pairs used for PCR were as follows: DPYSL3, forward, 5'-CCTCGGCATAGATGGAACC-3', and reverse, 5'-TCTGGGCAGTGCTGAAGGT-3'; runt-related transcription factor 2 (RUNX2), forward, 5'-GCCGGGAAT GATGAGAACTA-3', and reverse, 5'-GGACCGTCCACTGTC ACTTT-3'; (Osterix), forward, 5'-GGCGTCCTCCCTGCT TGA-3', and reverse, 5'-TGCTTTGCCCAGAGTTGTTG-3'; alkaline phosphatase (ALP), forward, 5'-GACAAGAAGCCC 
TTCACTGC-3', and reverse, 5'-AGACTGCGCCTGGTA GTTGT-3'; GAPDH, forward, 5'-TGAACGGGAAGCTCA CTGG-3', and reverse, 5'-TCCACCACCCTGTTGCTGTA-3'. The data were analyzed using the $2^{-\triangle \Delta C q}$ relative expression method (19). Each experiment was performed in triplicate.

Western blot analysis. Western blot analysis was performed as described previously (20). Protein extracts were prepared from ADSCs cells using RIPA buffer (Invitrogen; Thermo Fisher Scientific. Inc.) and protein concentration was determined using the BCA Protein Assay kit (Thermo Fisher Scientific, Inc.). Samples were combined with $2 X$ SDS loading buffer, boiled for $10 \mathrm{~min}$ and protein $(20 \mu \mathrm{g})$ was loaded onto a $10 \%$ or $4-20 \%$ gradient SDS-PAGE gel. Proteins were transferred to a PVDF membrane (EMD Millipore, Billerica, MA, USA) over $2 \mathrm{~h}$ at $350 \mathrm{~mA}$, and membranes were incubated in Odyssey Blocking Buffer (LI-COR Biosciences, Lincoln, NE, USA) for $2 \mathrm{~h}$ at room temperature. Following incubation with primary antibodies overnight at $4^{\circ} \mathrm{C}$, the blots were washed three times in TBS containing $0.1 \%$ Tween-20 for $15 \mathrm{~min}$ and then incubated with peroxidase- or IRDye-conjugated secondary antibody (1:5,000; cat. no. 4418; Cell Signaling Technology, Inc., Danvers, MA, USA) for $1 \mathrm{~h}$ in TBS, $0.1 \%$ Tween-20 at room temperature. Immunoreactivity was detected using an ECL reagent with a LI-COR imaging system. Primary antibodies were as follows: GAPDH (dilution, 1:1,000; cat. no. 8884; Cell Signaling Technology, Inc.), DPYSL3 (1:100; cat. no. sc-100323; Santa Cruz Biotechnology, Inc., Dallas, TX USA).

Alizarin red staining (ARS). ARS was performed at 14 days following osteogenic induction to detect the osteoblast calcification following the indicted treatments according to the manufacturer's instructions. Cells in 24-well plates were washed with PBS, fixed in 95\% ethanol for $10 \mathrm{~min}$ at room temperature, washed with distilled water, and stained at room temperature for $30 \mathrm{~min}$ using alizarin red solution $1 \mathrm{~g}$ Tris and $0.1 \mathrm{~g}$ alizarin red (Bio Basic Inc., Markham, ON, Canada) in $100 \mathrm{ml}$ ultrapure water]. Following washing with distilled water twice, the cells were photographed using an optical microscope.

ALP activity. An ALP assay kit (Beyotime Institute of Biotechnology, Hangzhou, China) was used to measure the ALP activity, according to the manufacturer's instructions. Total protein was extracted using radioimmunoprecipitation assay lysis buffer (included in the ALP assay kit) and the protein concentration was determined using a bicinchoninic acid assay. The cell lysate and substrate (obtained from the ALP assay kit) were added to a 96-well plate and incubated for $10 \mathrm{~min}$ at $37^{\circ} \mathrm{C}$. Following the addition of stop solution, the absorbance was determined at $405 \mathrm{~nm}$ using an ELISA microplate reader.

Statistical analysis. Quantitative data were reported as the mean \pm standard deviation. Statistical analysis was performed using one-way analysis of variance followed by a Bonferroni test for multiple groups, or a Student's t-test for the analysis of differences between two groups (using SPSS 13.0 software; SPSS, Inc., Chicago, IL, USA). P $<0.05$ was considered to indicate a statistically significant difference.

\section{Results}

DEMs and DEMis identification. Alterations in miRNA and mRNA expression profile during osteogenic differentiation were analyzed in human ADSCs using a microarray-based approach (GSE72429 and GSE37329). A total of 16 DEMis were observed to be important in this physiological process, while 185 DEMs were identified The predicted target genes were aligned with the DEMs and a number of DEMs were potentially regulated by $>1$ DEMis, according to the prediction result. These key genes are known to be involved in the osteogenic differentiation of ADSCs (Tables I and II). Among the DEMis, six miRNAs (miR-143-3p, miR-135a-5p, miR-31-5p, miR-22-3p, miR-193b-3p and let-7i-5p) were highly associated with the osteogenesis of ADSCs.

miRNA-mRNA and PPI networks. To speculate on the function of mRNAs targeted by miRNAs, a network among miRNAs and mRNAs was constructed and visualized. As shown in Fig. 1A, the miRNA-mRNA network consisted of 72 mRNA and nine miRNA nodes. Bioinformatics analysis highlighted six miRNAs among the network (miR-143-3p, miR-135a-5p, miR-31-5p, miR-22-3p, miR-193b-3p and let-7i-5p; Fig. 1B) that exhibited $>15$ predicted DEM targets. The PPI network of DEMs was illustrated in order to select significant genes (Fig. 1C).

Functional prediction of miRNAs based on the miRNA-mRNA network. To further understand the function and mechanism of identified DEMs and DEMis, functional and pathway enrichment analyses, including GO and KEGG, were performed using DAVID. For DEMs analysis, the results of GO analysis revealed 105 enriched GO terms in the 'Biological Process' category, particularly in the regulation of cytosolic calcium ion concentration, 16 enriched terms in the 'Molecular Function' category and 14 enriched terms in the in the 'KEGG pathway' category, according to $\mathrm{P}<0.05$ and Benjamini corrected $\mathrm{P}<0.05$ (Fig. 2A). The top significant terms in the Biological Processes, Molecular Function and KEGG pathway categories of DEMis are shown in Fig. 2B.

DPYSL3 downregulation in the process of the osteogenesis of ADSCs. In order to explore the possible target genes involved in the osteogenesis of ADSCs from the aforementioned miRNA-mRNA network, the mechanisms of miRNA mediation of downstream target genes were analyzed. It was identified that dihydropyrimidinase like 3 (DPYSL3) may be modulated by four differential DEMis, and was in the top 10 downregulated genes. Therefore DPYSL3 was selected as the target gene in the osteogenesis of ADSCs. Firstly, to verify the osteogenesis model of ADSCs, the expression of RUNX2, a marker of osteogenesis, was examined in ADSCs. It was observed that the expression of this gene was increased in the process of osteogenesis of ADSCs (Fig. 3A). RT-qPCR results indicated that the expression of DPYSL3 was decreased in the process of osteogenesis of ADSCs (Fig. 3B). Significant differences were observed following 7 and 14 days but not following 3 days of osteogenesis, when compared with the control group. 
Table I. List of identified DEMis.

\begin{tabular}{lcccc}
\hline miRNA & P-value & $\log _{2} \mathrm{FC}$ & Regulation & $\begin{array}{c}\text { Number of } \\
\text { targets DEMs }\end{array}$ \\
\hline miR-210 & 0.03826 & 5.27376 & $\mathrm{Up}$ & 0 \\
miR-143 & 0.00904 & 4.60661 & $\mathrm{Up}$ & 29 \\
miR-7 & 0.00275 & 4.16496 & $\mathrm{Up}$ & 0 \\
miR-100 & 0.00975 & 3.97587 & $\mathrm{Up}$ & 2 \\
let-7i & 0.00945 & 3.86616 & $\mathrm{Up}$ & 17 \\
miR-487a & 0.00953 & 3.82785 & $\mathrm{Up}$ & 3 \\
miR-502-3p & 0.00977 & 3.66819 & $\mathrm{Up}$ & 6 \\
miR-193b & 0.00961 & 3.55430 & $\mathrm{Up}$ & 9 \\
miR-22 & 0.00994 & 1.45696 & $\mathrm{Up}$ & 17 \\
miR-31 & 0.04184 & 1.03064 & Down & 0 \\
miR-642b & 0.03651 & -1.03150 & Down & 0 \\
miR-1181 & 0.00443 & -1.06189 & Down & 0 \\
miR-1275 & 0.01455 & -1.15712 & Down & 0 \\
miR-762 & 0.00403 & -1.39160 & Down & 21 \\
miR-135a & 0.02155 & -1.87443 & Down & 0 \\
miR-629 & 0.00609 & -5.71026 & & 0 \\
\hline
\end{tabular}

miRNA/miR, microRNA; DEMs, differentially expressed mRNAs; DEMis, differentially expressed miRNAs.

Table II. Expressed mRNAs targeted by miRNAs.

\begin{tabular}{|c|c|c|c|c|}
\hline Gene & Gene title & P-value & $\log _{2} \mathrm{FC}$ & Targeted by \\
\hline PPARGC1A & PPARG coactivator 1 alpha & 0.0036 & 2.35 & $\begin{array}{l}\text { let-7i-5p, miR-193b-3p, miR-31-5p, miR-22-3p, } \\
\text { miR-487a-3p }\end{array}$ \\
\hline DPYSL3 & Dihydropyrimidinase like 3 & 0.0173 & -2.07 & miR-143-3p, let-7i-5p, miR-22-3p, miR-31-5p, \\
\hline ADAMTS6 & $\begin{array}{l}\text { ADAM metallopeptidase with } \\
\text { thrombospondin type } 1 \text { motif } 6\end{array}$ & 0.0015 & -1.72 & $\begin{array}{l}\text { miR-143-3p, let-7i-5p, } \\
\text { miR-135a-5p, miR-22-3p }\end{array}$ \\
\hline CNR1 & Cannabinoid receptor 1 & 0.0067 & 1.69 & miR-143-3p, miR-487a-3p, let-7i-5p \\
\hline EDN1 & Endothelin 1 & 0.0002 & 2.40 & miR-143-3p, let-7i-5p miR-135a-5p \\
\hline FBN2 & Fibrillin 2 & 0.0028 & -1.68 & miR-143-3p, miR-22-3p miR-502-3p \\
\hline LGR4 & $\begin{array}{l}\text { Leucine rich repeat containing } \\
\text { G protein-coupled receptor } 4\end{array}$ & 0.0030 & -2.62 & let-7i-5p, miR-193b-3p miR-487a-3p \\
\hline NTNG1 & Netrin G1 & 0.0128 & -1.56 & miR-22-3p, miR-502-3p miR-135a-5p \\
\hline MMP16 & Matrix metallopeptidase 16 & 0.0225 & -2.90 & miR-135a-5p, miR-31-5p miR-193b-3p \\
\hline
\end{tabular}

miRNA/miR, microRNA.

Identified effects for silencing or overexpression of DPYSL3. To determine the functional role of DPYSL3 in the ADSC osteogenesis process, knockdown of DPYSL3 expression in CRC cells was achieved using two independent lentiviral-mediated short hairpin RNAs (shRNAs). Compared with the sh-GFP control, DPYSL3 levels in the sh-DPYSL3-1 and sh-DPYSL3-2 groups were significantly reduced (Fig. 3C). Furthermore, ectopic expression of DPYSL3 could increase DPYSL3 expression level. ADSCs were infected with lentiviral vectors containing DPYSL3. Overexpression of DPYSL3 was associated with increased DPYSL3 expression, both at the
mRNA and protein levels (Fig. 3D). These results indicate that the silencing or overexpression of DPYSL3 may decrease or increase the DPYSL3 levels, respectively.

DPYSL3 is the target gene in the process of osteogenesis of ADSCs. Further investigation into the functional role of DPYSL3 in the process of osteogenesis of ADSCs was performed. Following treatment with osteogenic medium for 14 days, it was observed that the silencing of DPYSL3 expression significantly promoted the increased expression of osteoblast differentiation-associated genes, RUNX2, OSX and ALP (Fig. 4A). By 
A
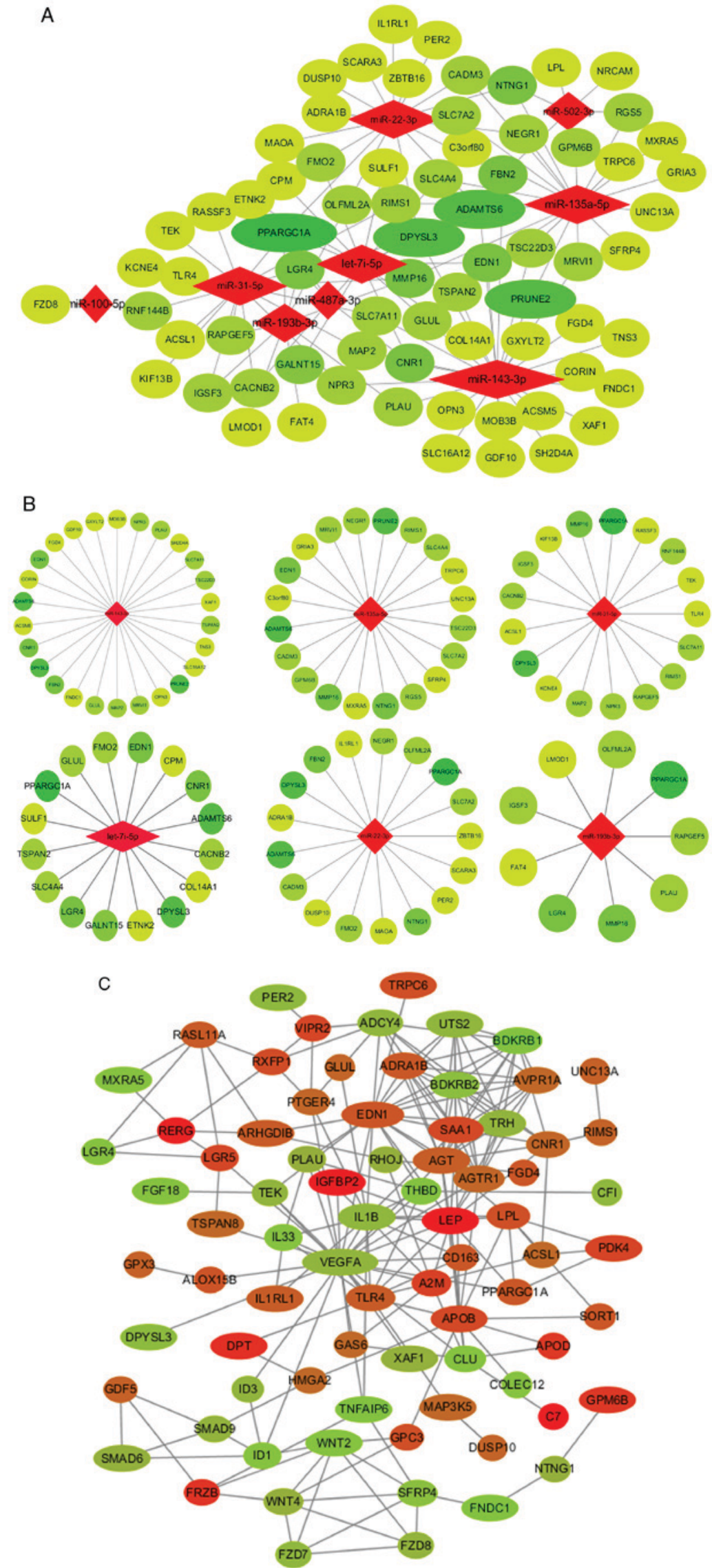

Figure 1. miRNA-mRNA network, PPI network and hub genes. (A) Core miRNA-mRNA network. Red diamonds represent miRNAs. Light green circles represent upregulated and dark green circles represent downregulated genes; the size of the circles reflects the fold change in mRNA expression following osteoblastic induction; the larger the size, the greater the fold change. (B) Six miRNAs (miR-143-3p, miR-135a-5p, miR-31-5p, let-7i-5p, miR-22-3p and miR-193b-3) and their potential target DEMs. Red diamonds represent miRNAs. Light green circles represent upregulated and dark green circles represent downregulated genes. (C) PPI network of DEMs. Red circles represent upregulated and green circles represent downregulated genes, the lighter the color, the greater the fold change. miRNA, microRNA; PPI, protein-protein interaction; DEMs, differentially expressed mRNAs. 

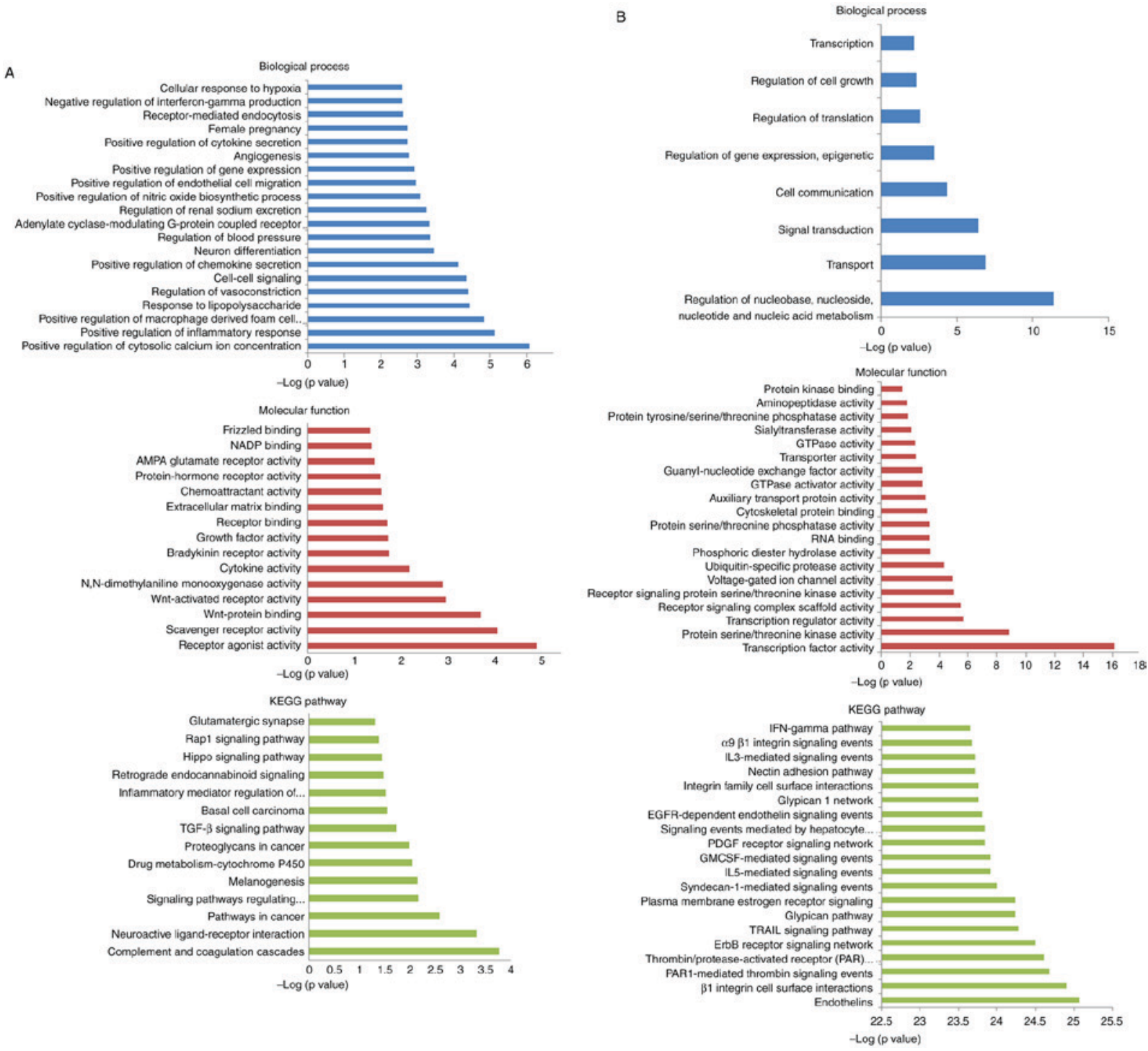

Figure 2. GO and KEGG pathway enrichment analysis. (A) GO and KEGG pathway enrichment analysis of DEMs, with the GO biological process (top), molecular function (middle) and KEGG pathway (bottom). (B) GO and KEGG pathway enrichment analysis of DEMis, with the GO biological process (top), molecular function (middle) and KEGG pathway (bottom). GO, Gene Ontology; KEGG, Kyoto Encyclopedia of Genes and Genomes; DEMs, differentially expressed mRNAs; DEMis, differentially expressed microRNAs.

contrast, overexpression of DPYSL3 significantly reduced the expression levels of these genes (Fig. 4B). Western blot analysis demonstrated that overexpression or silencing of DPYSL3 could decrease or increase the protein levels of RUNX2 following induction of differentiation using osteogenic medium for 14 days, respectively (Fig. 4C). Furthermore, ALP activity demonstrated a significant increase or decrease following silencing or overexpression of the DPYSL3 gene, respectively (Fig. 4D). These findings were supported by the ARS results at 14 days indicating the same tendency (Fig. 4E).

\section{Discussion}

MSCs are bone marrow stromal cells that are capable of differentiating into osteogenic, chondrogenic, adipogenic or myogenic cell lineages (21-23). Osteoblastic differentiation is a complex, closely regulated process that is critical for proper bone formation and is influenced by a variety of endogenous and environmental factors, including bone morphogenetic proteins, peroxisome proliferator-activated receptor $\gamma$, RUNX2 and Wnt/ $\beta$-Catenin $(24,25)$. However, its effect and the underlying mechanisms involved in the progression of bone regeneration remain unclear.

Developments in high-throughput technology have provided a large amount of osteogenesis profiles, which provide information that enables the investigation of osteoblastic differentiation. Bioinformatics analysis of the data has indicated that specific genes with aberrant expression may be important for osteoblastic differentiation or function as potential biomarkers for osteogenesis diagnosis and prognosis (26-29). In particular, miRNAs have been reported to be involved in the regulation of cellular behavior, including differentiation and development, metabolism, proliferation, apoptosis, viral infection and tumorigenesis (30-32). Dysregulation of miRNA expression may result in various pathological states (33). Recently, certain miRNAs were identified as regulators of post-transcriptional gene expression and were therefore considered important for osteogenesis $(34,35)$. 

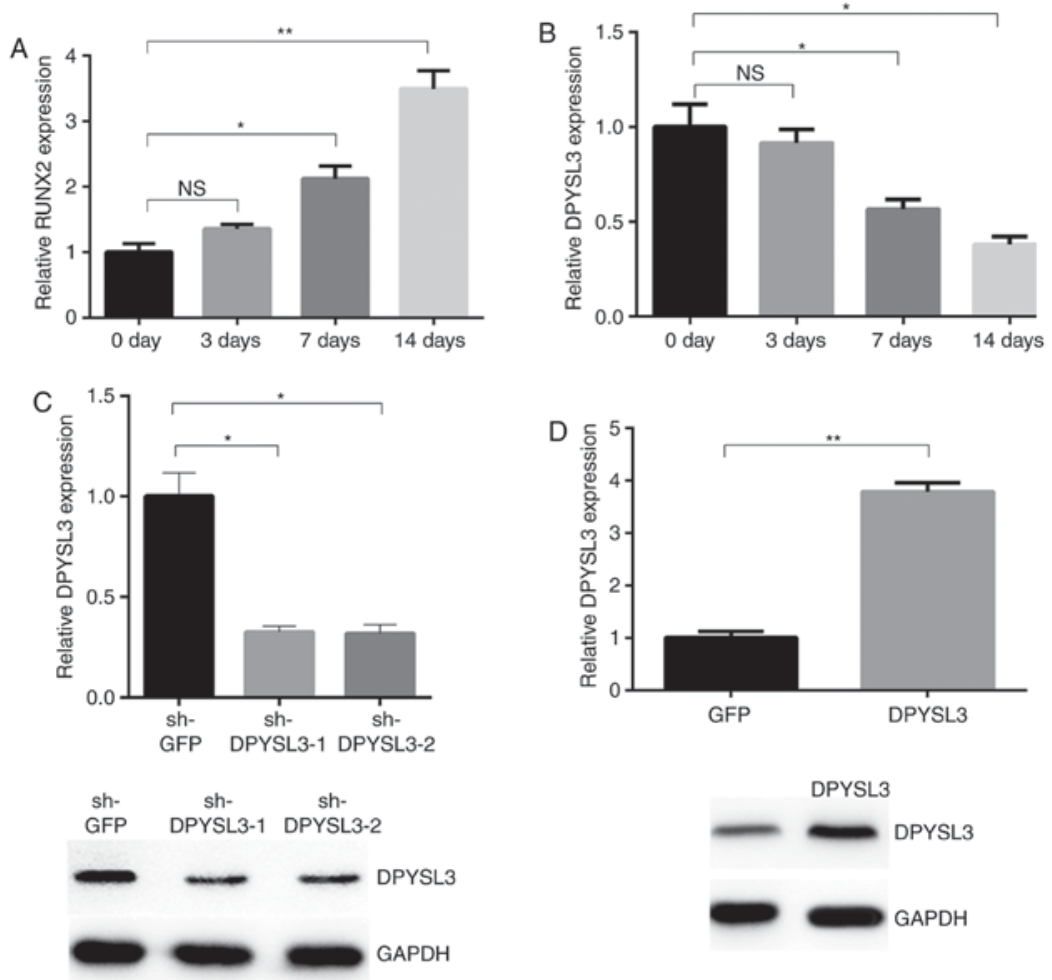

Figure 3. DPYSL3 downregulation in the process of the osteogenesis of ADSCs. (A) RUNX2 expression at different time points measured by RT-qPCR following osteoblastic induction. (B) DPYSL3 expression at different time points measured by RT-qPCR following osteoblastic induction. (C) RT-qPCR and western blot analysis of DPYSL3 expression in knockdown and control cells. (D) RT-qPCR and western blot analysis of DPYSL3 in overexpression and control cells. "P<0.05 and "** $\mathrm{P}<0.01$ vs. control group. DPYSL3, dihydropyrimidinase like 3; ADSCs, adipose-derived stem cells; RUNX2, runt-related transcription factor 2; RT-qPCR, reverse transcription-quantitative polymerase chain reaction; sh-, small hairpin.

A

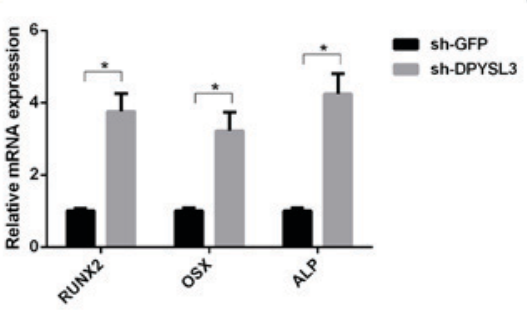

C

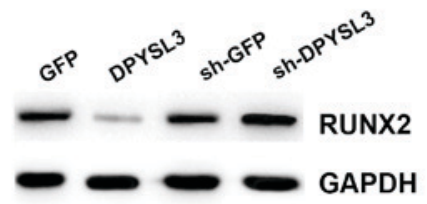

B

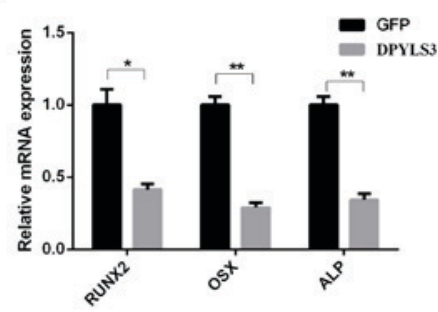

D

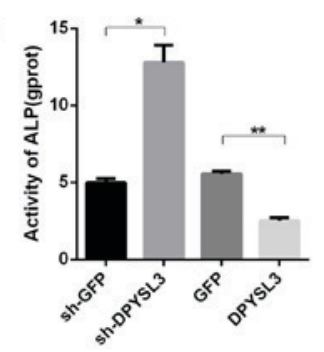

E

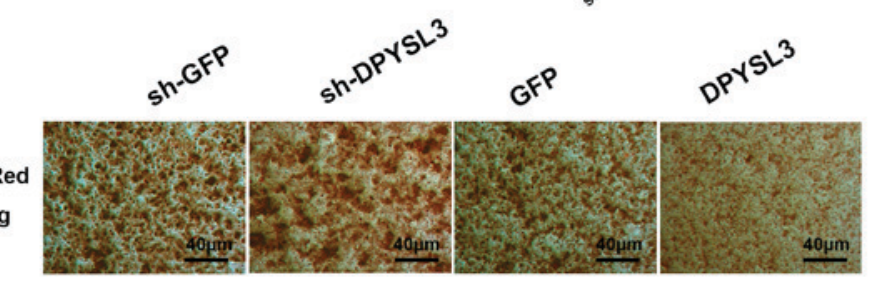

Figure 4. DPYSL3 inhibits osteoblastic differentiation. Human ADSCs were transfected with (A) DPYSL3 shRNA or (B) LV-DPYSL3, respectively for $48 \mathrm{~h}$, and the mRNA expression of osteoblastic differentiation-associated genes, RUNX2, OSX and ALP was determined by RT-qPCR. (C) Western blot analysis detecting RUNX2 expression in DPYSL3 silence or overexpression in ADSCs cells. (D) Alkaline phosphatase activity in the differentiated ADSCs. (E) Alizarin red staining of differentiated ADSCs. ${ }^{*} \mathrm{P}<0.05$ and ${ }^{* *} \mathrm{P}<0.01$ vs. the control group. DPYSL3, dihydropyrimidinase like 3; ADSCs, LV-DPYSL3, overexpressed dihydropyrimidinase like 3; adipose-derived stem cells; shRNA, short hairpin RNA; RUNX2, runt-related transcription factor 2; OSX, Osterix; RT-qPCR, reverse transcription-quantitative polymerase chain reaction; ALP, alkaline phosphatase; sh-, small hairpin. 
In previous studies, GO and pathway analyses were useful tools for analyzing biological functions, which were enriched among differentially expressed coding-genes $(36,37)$. GO analysis is used to probe the roles of differentially expressed genes, and annotate described genes and gene products distributed among all organisms. KEGG is a database resource that integrates genomic, chemical and systemic functional information; KEGG pathway analysis can provide promising and more biologically meaningful results, including information regarding molecular interactions and cellular processes (38). Gene catalogs from fully sequenced genomes are linked to higher-level systemic functions of the cell, the organism and the ecosystem $(39,40)$. In the GO analysis, the term of regulation of cytosolic calcium ion concentration ranks greatly statistics significance, proving the reliability of this study. In addition, the molecular function analysis of the Wnt-activated receptor and the Wnt-protein binding indicated significant involvement in the osteoblastic differentiation $(\mathrm{P}<0.05$, Benjamini corrected $\mathrm{P}<0.05)$. Pathway analysis further demonstrated that 14 pathways were enriched. The transforming growth factor beta and the ras-proximate-1 signaling pathway, as well as the signaling pathways regulating pluripotency of stem cells, have been observed to serve important roles in the osteogenesis of ADSCs $(41,42)$.

Emerging evidence has demonstrated that miRNAs have displayed superior potential as diagnostic and prognostic biomarkers due to their close association between mRNA expression and function $(43,44)$. Recently, previous studies have identified several miRNA-focused signatures that may advance the diagnoses of diseases, including ankylosing spondylitis, oral squamous cell carcinoma and multiple myeloma (45-47). However, the diagnostic role of miRNAs in the osteogenesis of ADSCs has not been fully investigated. In order to identify the key miRNAs involved, which may be used as potential novel biomarkers for the clinical diagnosis and treatment of the osteogenesis of ADSCs, the hub nodes and the number of relationship pairs were used in the present study. Previous studies have demonstrated that hub nodes, which are characterized by their high degree of connectivity to other nodes, may be important genes that could prove valuable to future study $(48,49)$. Generally, miRNAs with multiple relationship pairs take part in more mRNA interactions, thus identifying the miRNA as a hub. Hence, miRNAs are important in network organization (50). In the current study, six miRNAs (miR-143-3p, miR-135a-5p, miR-31-5p, miR-22-3p, miR-193b-3p and let-7i-5p) were identified as topological key nodes, where the node degrees and the number of miRNA-mRNA pairs were significantly higher compared with other miRNAs. This indicates that these miRNAs have profound implications for the osteogenesis of ADSCs and may be considered as key miRNAs.

Further analyses indicated that one of these identified genes, DPYSL3, was predicted to be modulated by the majority of miRNAs and exhibited a greater fold change. These findings indicate that DPYSL3 may be a critical gene involved in osteogenesis. However, the contribution of DPYSL3 to osteogenesis of ADSCs was not evaluated in the present study. By analyzing its expression in the process of osteogenesis of ADSCs, it was demonstrated that DPYSL3 expression was gradually decreased, which indicates that this gene might be involved in the osteogenesis of ADSCs. The selective knockdown or overexpression of this gene demonstrated that silencing of DPYSL3 promoted the expression of osteoblast differentiation-associated genes, while overexpression of DPYSL3 inhibited the expression of these genes following the induction by osteogenic medium for 14 days. Simultaneously, ALP activity exhibited a significant increase or decrease following silencing or overexpression of DPYSL3, respectively. The results were confirmed by ARS at 14 days, which demonstrated the same trend. These results indicated that DPYSL3 may be a target gene involved in the process of osteogenesis of ADSCs.

In conclusion, the miRNA-mRNA network constructed in the present study may enable the analysis of miRNA-mRNA-mediated genes in the development of osteogenesis of ADSCs at a system-wide level. These results identified DPYSL3 as a novel regulator in osteogenesis of ADSCs, and may provide novel insight into the potential of DPYSL3 as a therapeutic target in the management of bone regeneration-associated diseases.

\section{Acknowledgements}

The authors are grateful to Professor Jianjiang Zhao (Laboratory for Oral Diseases, Department of Oral and Maxillofacial Surgery, Stomatological Hospital, Southern Medical University, China) and Professor Qing Li (Guangzhou School of Clinical Medicine, Southern Medical University, Guangzhou General Hospital of Guangzhou Military Region, China) for providing help with the data analysis.

\section{Funding}

This study was financially supported by National Natural Science Foundation of China (81670950) and the Science and Technology Project of Guangdong Province (201802020018).

\section{Availability of data and materials}

The datasets used and/or analyzed during the current study are available from the corresponding author on reasonable request.

\section{Authors' contributions}

BJ, JZ and QL designed the experiments, BJ, ZZ and XQ carried out the experiments, BJ and XS analyzed the experimental results. $\mathrm{BJ}, \mathrm{HC}$ and $\mathrm{XZ}$ analyzed the sequencing data and developed the analytical tools, BJ and XQ wrote the manuscript, and JZ and QL contributed to finalizing the manuscript.

\section{Ethics approval and consent to participate}

Not applicable.

\section{Consent for publication}

Not applicable.

\section{Competing interests}

All authors declare that they have no competing interests. 


\section{References}

1. Pensak MJ and Lieberman JR: Gene therapy for bone regeneration. Curr Pharm Des 19: 3466-3473, 2013.

2. Thor A, Palmquist A, Hirsch JM, Rännar LE, Dérand P and Omar O: Clinical, morphological, and molecular evaluations of bone regeneration with an additive manufactured osteosynthesis plate. J Craniofac Surg 27: 1899-1904, 2016.

3. D'Mello S, Atluri K, Geary SM, Hong L, Elangovan S and Salem AK: Bone regeneration using Gene-Activated matrices. AAPS J 19: 43-53, 2017.

4. Kristjánsson B and Honsawek S: Mesenchymal stem cells for cartilage regeneration in osteoarthritis. World J Orthop 8: 674-680, 2017

5. Roura S, Gálvez-Montón C, Mirabel C, Vives J and Bayes-Genis A: Mesenchymal stem cells for cardiac repair: Are the actors ready for the clinical scenario? Stem Cell Res Ther 8: 238, 2017.

6. Manatsathit W, Samant $\mathrm{H}$ and Nakayuenyongsuk W: Mesenchymal stem cells for hepatitis B patients with acute on chronic liver failure-are we there? Hepatology 66: 1705-1706, 2017.

7. Moreira A, Kahlenberg S and Hornsby P: Therapeutic potential of mesenchymal stem cells for diabetes. J Mol Endocrinol 59: R109-R120, 2017.

8. Ding SLS, Kumar S and Mok PL: Cellular reparative mechanisms of mesenchymal stem cells for retinal diseases. Int J Mol Sci 18: pii: E1406, 2017

9. Roskies MG, Fang D, Abdallah MN, Charbonneau AM, Cohen N, Jordan JO, Hier MP, Mlynarek A, Tamimi F and Tran SD: Three-dimensionally printed polyetherketoneketone scaffolds with mesenchymal stem cells for the reconstruction of critical-sized mandibular defects. Laryngoscope 127 E392-E398, 2017.

10. Razmkhah M, Mansourabadi Z, Mohtasebi MA, Talei AR and Ghaderi A: Cancer and normal adipose-derived mesenchymal stem cells (ASCs): Do they have differential effects on tumor and immune cells? Cell Biol Int 42: 334-343, 2018.

11. Carstens MH, Mendieta M, Pérez C, Villareal E and Garcia R: Assisted salvage of ischemic fasciocutaneous flap using Adipose-Derived mesenchymal stem cells: In-Situ revascularization. Aesthet Surg J 37 (Suppl 3): S38-S45, 2017.

12. Gao W, Zhang L, Zhang Y, Sun C, Chen X and Wang Y: Adipose-derived mesenchymal stem cells promote liver regeneration and suppress rejection in small-for-size liver allograft. Transpl Immunol 45: 1-7, 2017.

13. Calabrese G, Giuffrida R, Forte S, Fabbi C, Figallo E, Salvatorelli L, Memeo L, Parenti R, Gulisano M and Gulino R: Human adipose-derived mesenchymal stem cells seeded into a collagen-hydroxyapatite scaffold promote bone augmentation after implantation in the mouse. Sci Rep 7: 7110, 2017

14. Ritchie ME, Phipson B, Wu D, Hu Y, Law CW, Shi W and Smyth GK: limma powers differential expression analyses for RNA-sequencing and microarray studies. Nucleic Acids Res 43 : e47, 2015.

15. Lewis BP, Burge CB and Bartel DP: Conserved seed pairing, often flanked by adenosines, indicates that thousands of human genes are microRNA targets. Cell 120: 15-20, 2005.

16. Dennis GJ Jr, Sherman BT, Hosack DA, Yang J, Gao W, Lane HC and Lempicki RA: DAVID: Database for annotation, visualization, and integrated discovery. Genome Biol 4: P3, 2003.

17. Sun C, Yuan Q, Wu D, Meng X and Wang B: Identification of core genes and outcome in gastric cancer using bioinformatics analysis. Oncotarget 8: 70271-70280, 2017.

18. Shannon P, Markiel A, Ozier O, Baliga NS, Wang JT, Ramage D, Amin N, Schwikowski B and Ideker T: Cytoscape: A software environment for integrated models of biomolecular interaction networks. Genome Res 13: 2498-2504, 2003.

19. Livak KJ and Schmittgen TD: Analysis of relative gene expression data using real-time quantitative PCR and the 2(-Delta Delta C(T)) method. Methods 25: 402-408, 2001.

20. Chu H, Jia B, Qiu X, Pan J, Sun X, Wang Z and Zhao J: Investigation of proliferation and migration of tongue squamous cell carcinoma promoted by three chemokines, MIP-3 $\alpha$, MIP-1 $\beta$, and IP-10. Oncotargets Ther 10: 4193-4203, 2017.

21. He BC, Chen L, Zuo GW, Zhang W, Bi Y, Huang J, Wang Y, Jiang W, Luo Q, Shi Q, et al: Synergistic antitumor effect of the activated PPARgamma and retinoid receptors on human osteosarcoma. Clin Cancer Res 16: 2235-2245, 2010.
22. Aubin JE: Regulation of osteoblast formation and function. Rev Endocr Metab Disord 2: 81-94, 2001.

23. Deng ZL, Sharff KA, Tang N, Song WX, Luo J, Luo X, Chen J, Bennett E, Reid R, Manning D, et al: Regulation of osteogenic differentiation during skeletal development. Front Biosci 13: 2001-2021, 2008

24. Wagner ER, He BC, Chen L, Zuo GW, Zhang W, Shi Q, Luo Q, Luo X, Liu B, Luo J, et al: Therapeutic implications of PPARgamma in human osteosarcoma. PPAR RES 2010: 956427, 2010.

25. Tang N, Song WX, Luo J, Haydon RC and He TC: Osteosarcoma development and stem cell differentiation. Clin Orthop Relat Res 466: 2114-2130, 2008.

26. Zhao W, Wang D, Zhao J and Zhao W: Bioinformatic analysis of retinal gene function and expression in diabetic rats. Exp Ther Med 14: 2485-2492, 2017.

27. Koehler AV, Korhonen PK, Hall RS, Young ND, Wang T, Haydon SR and Gasser RB: Use of a bioinformatic-assisted primer design strategy to establish a new nested PCR-based method for Cryptosporidium. Parasit Vectors 10: 509, 2017.

28. Zhang C, Chen Y, Mao X, Huang Y, Jung SY, Jain A, Qin J and Wang Y: A bioinformatic algorithm for analyzing cell signaling using temporal proteomic data. Proteomics 17, 2017. doi: 10.1002/pmic.201600425

29. Tsukanov KY, Krasnenko AY, Plakhina DA, Korostin DO, Churov AV, Druzhilovskaya OS, Rebrikov DV and Ilinsky VV: A bioinformatic pipeline for NGS data analysis and mutation calling in human solid tumors. Biomed Khim 63: 413-417, 2017 (In Russian).

30. Irwandi RA and Vacharaksa A: The role of microRNA in periodontal tissue: A review of the literature. Arch Oral Biol 72: 66-74, 2016.

31. Huang Y, Shen XJ, Zou Q, Wang SP, Tang SM and Zhang GZ: Biological functions of microRNAs: A review. J Physiol Biochem 67: 129-139, 2011.

32. Hamidi-Asl E, Palchetti I, Hasheminejad E and Mascini M: A review on the electrochemical biosensors for determination of microRNAs. Talanta 115: 74-83, 2013.

33. Jia HY, Chen F, Chen JZ, Wu SS, Wang J, Cao QY, Chen Z and Zhu HH: MicroRNA expression profiles related to early stage murine concanavalin A-induced hepatitis. Cell Physiol Biochem 33: 1933-1944, 2014.

34. Khraiwesh B, Zhu JK and Zhu J: Role of miRNAs and siRNAs in biotic and abiotic stress responses of plants. Biochim Biophys Acta 1819: 137-148, 2012

35. van Wijnen AJ, van de Peppel J, van Leeuwen JP, Lian JB, Stein GS, Westendorf JJ, Oursler MJ, Im HJ, Taipaleenmäki H, Hesse E, et al: MicroRNA functions in osteogenesis and dysfunctions in osteoporosis. Curr Osteoporos Rep 11: 72-82, 2013.

36. Song C, Zhang J, Liu Y, Pan H, Qi HP, Cao YG, Zhao JM, Li S, Guo J, Sun HL and Li CQ: Construction and analysis of cardiac hypertrophy-associated lncRNA-mRNA network based on competitive endogenous RNA reveal functional lncRNAs in cardiac hypertrophy. Oncotarget 7: 10827-10840, 2016.

37. Wu Q, Guo L, Jiang F, Li L, Li Z and Chen F: Analysis of the miRNA-mRNA-lncRNA networks in ER+ and ER-breast cancer cell lines. J Cell Mol Med 19: 2874-2887, 2015.

38. Kanehisa M and Goto S: KEGG: Kyoto encyclopedia of genes and genomes. Nucleic Acids Res 28: 27-30, 2000.

39. Kanehisa M: Enzyme annotation and metabolic reconstruction using KEGG. Methods Mol Biol 1611: 135-145, 2017.

40. Kanehisa M, Furumichi M, Tanabe M, Sato Y and Morishima K: KEGG: New perspectives on genomes, pathways, diseases and drugs. Nucleic Acids Res 45 (D1): D353-D361, 2017.

41. Wu Y, Zhou J, Li Y, Zhou Y, Cui Y, Yang G and Hong Y: Rap1A regulates osteoblastic differentiation via the ERK and p38 mediated signaling. PLoS One 10: e143777, 2015.

42. Wu M, Chen G and Li YP: TGF- $\beta$ and BMP signaling in osteoblast, skeletal development, and bone formation, homeostasis and disease. Bone Res 4: 16009, 2016.

43. Bartel DP: MicroRNAs: Genomics, biogenesis, mechanism, and function. Cell 116: 281-297, 2004.

44. He L and Hannon GJ: MicroRNAs: Small RNAs with a big role in gene regulation. Nat Rev Genet 5: 522-531, 2004.

45. Shen X, Zhang Y, Wu X, Guo Y, Shi W, Qi J, Cong H, Wang X, $\mathrm{Wu} \mathrm{X}$ and Ju S: Upregulated lncRNA-PCAT1 is closely related to clinical diagnosis of multiple myeloma as a predictive biomarker in serum. Cancer Biomark 18: 257-263, 1017. 
46. Li X, Chai W, Zhang G, Ni M, Chen J, Dong J, Zhou Y, Hao L, Bai Y and Wang Y: Down-Regulation of IncRNA-AK001085 and its influences on the diagnosis of ankylosing spondylitis. Med Sci Monit 23: 11-16, 2017

47. Tang H, Wu Z, Zhang J and Su B: Salivary lncRNA as a potential marker for oral squamous cell carcinoma diagnosis. Mol Med Rep 7: 761-766, 2013.

48. Zhou M, Diao Z, Yue X, Chen Y, Zhao H, Cheng L and Sun J: Construction and analysis of dysregulated lncRNA-associated ceRNA network identified novel lncRNA biomarkers for early diagnosis of human pancreatic cancer. Oncotarget 7: 56383-56394, 2016

49. Zhang Y, Xu Y, Feng L, Li F, Sun Z, Wu T, Shi X, Li J and Li X: Comprehensive characterization of lncRNA-mRNA related ceRNA network across 12 major cancers. Oncotarget 7: 64148-64167, 2016.
50. Cao Y, Wang P, Ning S, Xiao W, Xiao B and Li X: Identification of prognostic biomarkers in glioblastoma using a long non-coding RNA-mediated, competitive endogenous RNA network. Oncotarget 7: 41737-41747, 2016.

(7)(9) This work is licensed under a Creative Commons Attribution-NonCommercial-NoDerivatives 4.0 International (CC BY-NC-ND 4.0) License. 